\title{
Characterization of the "simple-liquid" state in a polymeric system: Coherent and incoherent scattering functions
}

\author{
A. Arbe ${ }^{1, *}$ and J. Colmenero ${ }^{1,2}$ \\ ${ }^{1}$ Centro de Física de Materiales (CSIC-UPV/EHU)-Materials Physics Center (MPC), Apartado 1072, 20080 San Sebastián, Spain \\ ${ }^{2}$ Donostia International Physics Center, Paseo Manuel de Lardizabal 4, 20018 San Sebastián, Spain
}

(Received 12 June 2009; published 14 October 2009)

\begin{abstract}
By means of time-of-flight neutron scattering, we have characterized the dynamic structure factor and the hydrogen motions of polyethylene above its melting point. As signatures of simple-liquid dynamics, we observe that (i) in the explored dynamic window, the intermediate scattering functions display a single-step decay, without reminiscence of cage effects, and (ii) the structural relaxation as observed at the intermolecular structure factor peak shows a weak temperature dependence, indicating a low degree of interchain cooperativity. However, stretched functional forms are observed for all length scales and temperatures investigated. An apparent direct crossover from the microscopic regime to Rouse-like dynamics suggests an essential role of connectivity in the observed stretching. Finally, while at momentum-transfer values above the structure factor peak the relation between coherent and incoherent characteristic times is reasonably described by the de Gennes narrowing, at larger length scales it is not reproduced by any existing approach.
\end{abstract}

DOI: 10.1103/PhysRevE.80.041805

\section{INTRODUCTION}

Nowadays, it is well known that a signature of supercooled liquid state is a "two-step decay" of any correlation function. A first microscopic regime followed by a decaging toward the structural relaxation is the well-known picture proposed by the theoretical approach of the mode coupling theory [1] to the glass transition phenomenon. Also, two distinct regimes in the relaxation functions are considered by the coupling model (see, e.g., [2]). This behavior has been found in different systems including, e.g., low molecular weight systems, polymers, and colloids, either experimentally or by molecular-dynamics (MD) simulations (see, e.g., [3]).

Increasing temperature, the structural relaxation time decreases and approaches the microscopic time range. Hence, it is expected that at high-enough temperature, the intermediate decaging region would disappear. Then, the importance of cooperativity effects would be negligible and a simple liquidlike dynamics should be observed with an associated single decay of the correlations. This kind of dynamics would take place in a glass-former well above the melting point $T_{M}$. The questions arise: is it possible in some way to infer from this liquid state that the system is willing to form a glass at lower temperatures (i.e., does it still show similarities with the supercooled liquid state below the melting)? Or is the behavior of such a liquid as simple as the expected one, e.g., for a monoatomic liquid?

Studies on low-molecular weight glass-forming systems (glycerol, propylene carbonate, and benzophenone) by dielectric spectroscopy and depolarized light scattering well above their melting points do not reveal a transition to exponential behavior [4]. The authors concluded that the existence of the second stretched regime is a universal feature of molecular glass formers even well above $T_{M}$. They also pointed out that the relaxation stretching is clearly different depending on the technique used.

\footnotetext{
*a.arbe@ehu.es
}

PACS number(s): 61.41.+e, 64.70.pj, 61.05.fg

The situation with polymers is even more complicated. On one hand, most polymers cannot crystallize, i.e., no melting temperature can be properly identified in most cases. On the other hand, chain connectivity plays a major role, which signatures in the liquid state are not yet clear. We may then ask the question: what is the liquid state of a polymer? To give definite answers, a direct observation of the structural relaxation at a molecular level is mandatory. This is achieved by means of neutron-scattering experiments on the dynamic structure factor at the intermolecular peak, i.e., by following the decay of the correlations between the structural units in the system.

With these ideas in mind, we have used quasielastic neutron scattering to characterize at molecular level and with space-time resolution the dynamics of the chemically simplest polymeric liquid: polyethylene (PE). With measurements on a fully deuterated sample, we have investigated the dynamic structure factor (coherent scattering), revealing at intermolecular level the structural relaxation. A time-of-flight (TOF) spectrometer was used. This kind of instruments explore momentum-transfer $(Q)$ regions roughly between $\approx 0.3$ and $2 \AA^{-1}$ - where polymers usually present their intermolecular structure factor peak - and time scales in the picosecond region-where liquidlike dynamics is expected to occur. Moreover, using a protonated sample, we have accessed the self part of the van Hove correlation function for hydrogens (incoherent scattering). As our aim is to determine to which extent the dynamics of a liquid polymer can be considered as corresponding to a "simple liquid," one key point is to check the relationship between coherent and incoherent scattering functions in simple liquids predicted by de Gennes [5].

Due to its chemical simplicity, PE has been subject of a large number of neutron-scattering experiments. Probably due to the difficult interpretation of collective dynamics, almost all of them address incoherent scattering on protonated samples (see, e.g., [6-12]). To our knowledge, only two works have been published on the dynamic structure factor of PE $[7,9]$. None of them considered the here-raised questions: Buchenau et al. [7] did not analyze the spectral shape 
of the dynamic structure factor and no direct comparison was made between incoherent and coherent scatterings. Holzer et al. [9] focused on the semicrystalline regime.

On the other hand, polyethylene is also the ideal polymer to perform MD simulations (see, e.g., [13-20]). In some of those works $[16,18-20]$, the simulations have been combined with incoherent-neutron scattering measurements. The collective dynamics of molten PE and its relation with the selfmotions has never been addressed in those studies.

This paper is structured as follows. In Sec. II, we introduce the magnitudes measured by neutron scattering and present the experimental details of this work. Section III summarizes the main features established during the last years for the dynamics of glass-forming polymers below the melting point and briefly presents the Rouse model describing the single chain dynamics of polymer melts. The aim is to set the reference for further discussion of PE results in the liquid state. The two next sections are devoted to present the results and explain the procedure for data analysis. In the discussion (Sec. VI), we first address the structural information that can be extracted from the coherent data (Sec. VI A) and then we focus on the dynamical aspects (Sec. VI B). They include the observation of the structural relaxation (Sec. VI B 1) and of the H motions (Sec. VI B 2). The role played by chain connectivity is then considered in Sec. VI B 3. The comparison between collective and self-motions is discussed in Sec. VI B 4 and finally the main conclusions are summarized.

\section{EXPERIMENTAL}

\section{A. Samples}

We investigated fully protonated polyethylene (PEh) and fully deuterated polyethylene (PEd). The thickness was 0.12 $\mathrm{mm}$ for PEh and $0.7 \mathrm{~mm}$ for PEd, leading to neutron transmissions around $92 \%$. The molecular weight of both samples was $M_{w}=80 \mathrm{Kg} / \mathrm{mol}$ with polydispersities close to 2 in both cases. A melting temperature of $400 \mathrm{~K}$ was determined by differential scanning calorimetry (DSC).

\section{B. Neutron scattering}

The neutron intensity scattered into a solid angle between $\Omega$ and $\Omega+d \Omega$, after having exchanged an energy between $E$ and $E+d E$ with the sample can be expressed as (see, as general references, [21-23])

$$
\begin{aligned}
\frac{\partial^{2} \sigma}{\partial \Omega \partial E}= & \left(\frac{\partial^{2} \sigma}{\partial \Omega \partial E}\right)_{i n c}+\left(\frac{\partial^{2} \sigma}{\partial \Omega \partial E}\right)_{c o h}=\frac{\sigma_{i n c}}{4 \pi} S_{i n c}(Q, \omega) \\
& +\frac{\sigma_{c o h}}{4 \pi} S_{c o h}(Q, \omega) .
\end{aligned}
$$

Here, $\sigma_{\text {inc }}\left(\sigma_{\text {coh }}\right)$ is the incoherent (coherent) scattering cross section and $S_{\text {inc }}(Q, \omega)\left[S_{\text {coh }}(Q, \omega)\right]$ the incoherent (coherent) scattering function. $Q$ is the modulus of the momentum transfer and $\omega=E / \hbar$. For small energy transfers, the value of $Q$ is close to that corresponding to the elastic condition $Q_{e l}$, $Q_{e l}=4 \pi \sin (\theta / 2) / \lambda$, where $\theta$ is the scattering angle and $\lambda$ the wavelength of the incoming neutrons.
In samples containing different kinds of nuclei $\alpha$ (e.g., $\alpha$ : $\mathrm{H}, \mathrm{C}, \mathrm{O}, \mathrm{D}, \ldots)$ with relative abundance $n_{\alpha}, \sigma_{\text {inc }}=\Sigma_{\alpha} n_{\alpha} \sigma_{\text {inc }}^{\alpha}$ and $\sigma_{c o h}=\sum_{\alpha} n_{\alpha} \sigma_{c o h}^{\alpha}$, where $\sigma_{c o h}^{\alpha}=4 \pi b_{\alpha}^{2}\left(b_{\alpha}\right.$ : scattering length of nucleus $\alpha$ ). Due to the high value of $\sigma_{i n c}^{\mathrm{H}}(80.27$ barns $)$, the intensity scattered by protonated samples such as $\mathrm{PEh}$ is dominated by the incoherent contribution from the hydrogens $\quad\left[\sigma_{i n c}^{\mathrm{C}}=0, \sigma_{c o h}^{\mathrm{H}}=1.76\right.$ barns $\left(b_{\mathrm{H}}=-3.74 \mathrm{fm}\right), \sigma_{c o h}^{\mathrm{C}}$ $=5.56$ barns $\left.\left(b_{\mathrm{C}}=6.65 \mathrm{fm}\right)\right] . S_{\text {inc }}(Q, \omega)$ is the Fourier transform of the intermediate incoherent scattering function $S_{\text {inc }}(Q, t)$ and the double Fourier transform of $S_{\text {inc }}(Q, \omega)$ yields the self part of the van Hove correlation function $G_{\text {self }}(r, t)$. Incoherent scattering looks at correlations between the positions of the same nucleus at different times. For some simple cases, $G_{\text {self }}(r, t)$ is a Gaussian function $[21,23]$ and $S_{\text {inc }}(Q, t)$ is entirely determined by the mean squared displacement $\left\langle r^{2}(t)\right\rangle$,

$$
S_{\text {inc }}^{\text {gauss }}(Q, t)=\exp \left[-\frac{\left\langle r^{2}(t)\right\rangle}{6} Q^{2}\right] .
$$

In general, deviations from the Gaussian form of $G_{\text {self }}(r, t)$ [and consequently from Eq. (2)] are expected. $S_{\text {inc }}(Q, t)$ can then be expressed in terms of its expansion in $Q^{2}[24,25]$

$$
S_{\text {inc }}(Q, t)=\exp \left[-\frac{\left\langle r^{2}(t)\right\rangle}{6} Q^{2}+\frac{\alpha_{2}(t)\left\langle r^{2}(t)\right\rangle^{2}}{72} Q^{4}+\cdots\right],
$$

where the so-called second-order non-Gaussian parameter $\alpha_{2}(t)$ gives the leading correction.

On the other hand, since $\sigma_{i n c}^{D}=2.05$ barns and $\sigma_{c o h}^{D}$ $=5.59$ barns $\left(b_{D}=6.67 \mathrm{fm}\right)$, a perdeuterated sample such as PEd scatters mainly coherently-the incoherent contribution of D in PEd only amounts to less than $20 \%$ of the total scattering. Coherent scattering deals with relative positions of atomic pairs

$\left(\frac{\partial^{2} \sigma}{\partial \Omega \partial E}\right)_{c o h} \propto\left\langle\sum_{\alpha, \beta} \sum_{i \alpha, j \beta}^{N_{\alpha}, N_{\beta}} b_{\alpha} b_{\beta} \frac{1}{2 \pi \hbar} \int_{-\infty}^{\infty} e^{-i \omega t} e^{i \vec{Q}\left[\vec{r}_{i \alpha}(t)-\vec{r}_{j \beta}(0)\right]} d t\right\rangle$,

where $\alpha, \beta$ refer to the different kinds of isotopes. As the scattering lengths of $\mathrm{C}$ and $\mathrm{D}$ are nearly identical, coherent scattering in samples such as PEd delivers the true or total dynamic structure factor. For $t=0$ (equivalently, the integral over all energies), this corresponds to the total static structure factor $S(Q)$.

\section{MIBEMOL}

In this work, we used the TOF spectrometer G6-2 MIBEMOL at the Laboratoire Léon Brillouin (LLB), in Saclay (France). With an incident wavelength $\lambda=5.0 \AA$, an energy resolution $\delta E=92 \mu \mathrm{eV}$ (half width at half maximum, HWHM) was achieved and a $Q$ range of $0.51-2.23 \AA^{-1}$ was covered for elastic scattering.

The samples were placed into flat aluminum containers and put at an angle of $135^{\circ}$ with respect to the incident beam in order to avoid self-shielding and self-absorption effects at low- and intermediate-scattering angles. The high value of 
the transmission of the sample $(\approx 92 \%)$ allows neglecting multiple-scattering effects. The measuring times were of $\approx 4.5 \mathrm{~h}$ for the protonated sample and $6 \mathrm{~h}$ for the deuterated sample. We investigated the following temperatures above the melting: 421, 446, 477, 510, and $551 \mathrm{~K}$. Spectra at $180 \mathrm{~K}$ in the semicrystalline state were also recorded. Wavelengthdependent detector efficiency corrections were performed with the program SQOMEG available at the LLB by calibration with a vanadium measurement; these data were also employed as resolution function. The contribution from the sample container was determined at two temperatures (200 and $520 \mathrm{~K}$ ) and properly subtracted from the spectra. For the protonated sample, where a smooth $Q$ dependence is expected, data corresponding to different detectors were grouped to improve statistics, resulting in nine grouped spectra. For the deuterated sample, all detectors were considered independently in order to properly resolve the $Q$ dependence of the relevant parameters. The configuration of the detectors leads to a gap in the $Q$ range between 0.6 and $0.8 \AA^{-1}$ that becomes apparent in these results.

\section{GENERAL FRAMEWORK FOR GLASS-FORMING POLYMER DYNAMICS}

Neutron scattering investigations of the dynamics in glass-forming polymers in the supercooled liquid regime have demonstrated that the correlation functions decay in two steps. The microscopic one below $\approx 1-2$ ps is dominated by the fast motions within the cage imposed by the neighboring atoms and the correlation functions follow an approximately Debye-like behavior [26-29]. Above the picosecond, the second slow decay of both coherent and incoherent intermediate scattering functions shows stretched behavior and can be well described by stretched exponentials or Kohlrausch-Williams-Watts (KWW) functions

$$
S(Q, t)=A \exp \left[-\left(\frac{t}{\tau_{w}}\right)^{\beta}\right] .
$$

Here, $A$ is an amplitude factor, $\tau_{w}$ is the characteristic time, and $\beta$ is the shape parameter or stretching exponent which quantifies the deviation from simple exponential behavior. For coherent scattering, this has been proven by neutron spin-echo (NSE) measurements mainly at the intermolecular correlation peak in a large number of deuterated polymers [30]. Those studies have established the essential properties of the structural relaxation, such as the above-mentioned stretching and the scaling with the macroscopic viscosity, implying time-temperature superposition [30]. NSE [30] and backscattering (see, e.g., $[31,32]$ ) studies on the self-motions of $\mathrm{H}$ atoms in the range $Q \approx 0.2 \ldots 1 \AA^{-1}$ have shown that within the uncertainties, (i) the $\beta$ parameter is $Q$ independent and close to 0.5 , (ii) the characteristic time follows a $Q$ dependence $\tau_{w} \propto Q^{-2 / \beta}$, and (iii) the Lamb-Mössbauer factor $A$ is described by $A=\exp \left[-\left\langle u^{2}\right\rangle Q^{2} / 3\right]$. Such observations imply that the Gaussian approximation [Eq. (2)] is fulfilled [31,32], with $\left\langle r^{2}(t)\right\rangle \propto t^{\beta}$ (sublinear anomalous diffusion). However, weaker $Q$ dependences of the characteristic time are found at larger $Q$ values [33-43]. MD simulations on some polymers $[36,38]$ have allowed attributing these deviations to the non-
Gaussian events taking place in the decaging time region. There, the second-order non-Gaussian parameter $\alpha_{2}$ exhibits a main peak and the mean squared displacement of the atoms displays a kind of plateau connecting the microscopic regime below $\approx 1 \mathrm{ps}$ and the sublinear increase. At the structural relaxation time $\tau_{\alpha}$ (the characteristic time of the decay of the intermolecular correlations), the sublinear Gaussian regime of the atoms is already well established [34,44].

On the other hand, in the high-temperature range, the chain dynamics becomes very fast and may contribute to the neutron spectra. The Rouse model $[45,46]$ has proven to describe well [30] the chain dynamics at long times and intermediate-length scales, where the conformational entropy is the essential ingredient. This model starts from a Gaussian chain representing a coarse-grained polymer model where springs stand for the entropic forces between hypothetic beads. The chain consists of $N$ beads of length $\ell$. The solution of the corresponding Langevin equation (Brownian coupled oscillators) results in a spectrum of normal modes of characteristic times

$$
\tau_{p}=\frac{1}{4 W \sin ^{2} \frac{p \pi}{2 N}} .
$$

Here, $p=0 \ldots N-1$ is the mode number. The Rouse frequency $W$ is defined as the ratio between the entropic force $3 k_{B} T / \ell^{2}$ $\left(k_{B}\right.$ : Boltzmann constant) and the friction coefficient $\zeta$. The mean-square displacement of segment $n$ along the chain can be obtained as

$$
\begin{aligned}
\left\langle r_{n}^{2}(t)\right\rangle_{\text {Rouse }}= & 6 D_{R} t+\frac{4 N \ell^{2}}{\pi^{2}} \sum_{p=1}^{N} \frac{1}{p^{2}} \cos ^{2}\left(\frac{p \pi n}{N}\right) \\
& \times\left[1-\exp \left(-\frac{t}{\tau_{p}}\right)\right] .
\end{aligned}
$$

For small-enough $p$, one obtains

$$
\left\langle r^{2}(t)\right\rangle_{\text {Rouse }}^{p \ll N-1}=2 \sqrt{\frac{W \ell^{4}}{\pi}} t+6 D_{R} t .
$$

The self-correlation function $S_{\text {inc }}^{\text {Rouse }}(Q, t)$ is obtained in Gaussian approximation [Eq. (2)]. For $Q$ values where the diffusion contribution is not important, it has just a KWW form [Eq. (5)] with $\beta=0.5$ and characteristic time

$$
\tau_{\text {self }}^{\text {Rouse }}(Q, T)=\frac{9 \pi}{W \ell^{4}} Q^{-4} .
$$

Due to the patent similarities of the descriptions of the incoherent scattering functions in the $\alpha$-relaxation regime and in the Rouse regime, it is difficult to distinguish between these two dynamical regimes and elucidate the existence of a crossover between them. This question has recently been experimentally addressed in some polymers. In the case of poly(vinyl ethylene), the size of the "Rouse blob" (below which the model fails) was found to be about ten monomers or 20 bonds [44]. This size could be smaller in the case of polyisoprene [47]. Above its melting point, polyethylene oxide apparently shows an almost perfect Rouse behavior [48-50]; however, simulation results point to deviations oc- 

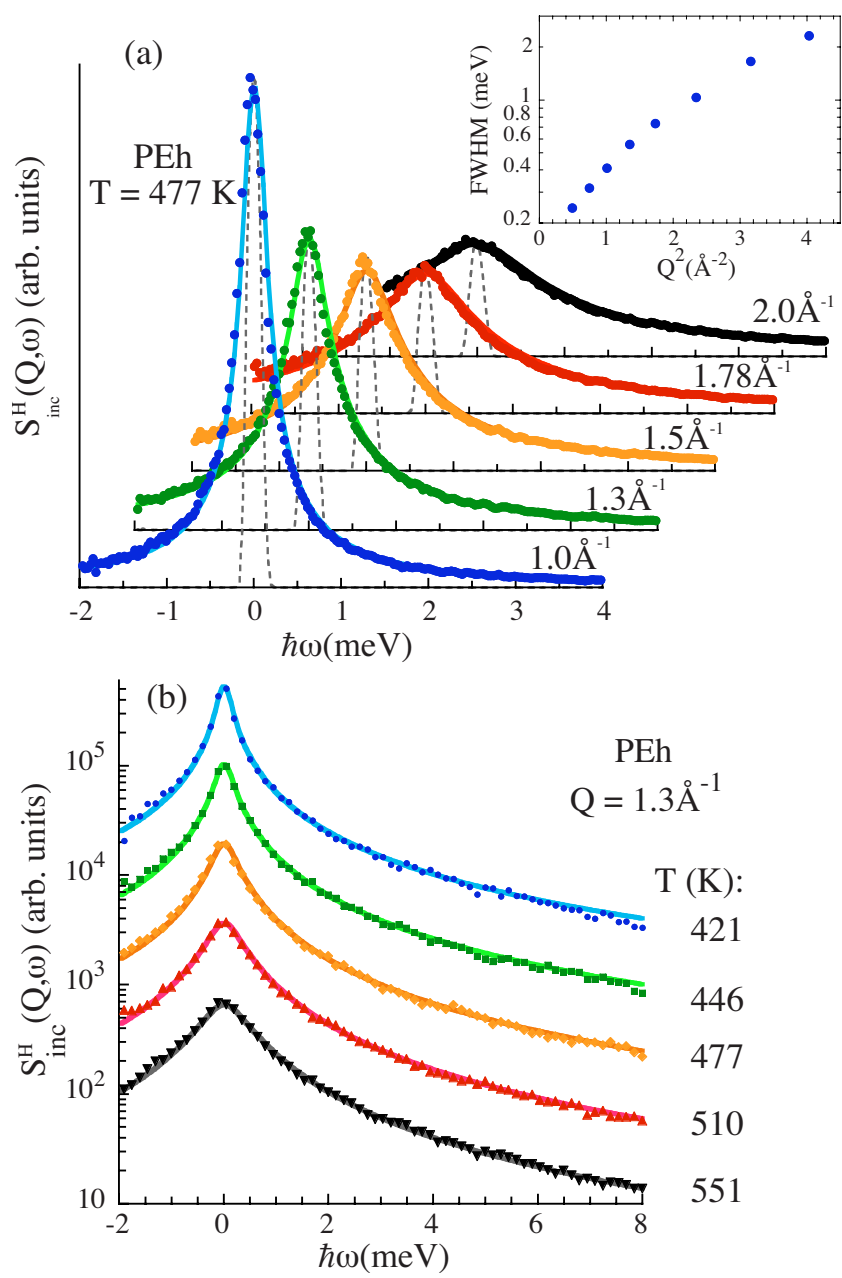

FIG. 1. (Color online) Spectra obtained by interpolation at constant $Q$ for the protonated sample: (a) at $477 \mathrm{~K}$ and the $Q$ values indicated; (b) at $1.3 \AA^{-1}$ for the different temperatures investigated in a logarithmic representation. In (b), the following factors have been applied for clarity: 16 (421 K), 4 (446 K), 1/4 (510 K), and $1 / 16(551 \mathrm{~K})$. Solid lines through the points are fits with KWW functions. Dotted lines in (a) show the resolution function. Insert in (a) shows the $Q$ dependence of the FWHM of the spectra at $477 \mathrm{~K}$.

curring at large mode numbers that, in addition to considering local stiffness of the chain, require assuming a significant increase of the mode friction for such mode numbers. A similar effect was also observed experimentally for polyisobutylene $[51,52]$.

\section{RESULTS}

\section{A. Protonated sample: Incoherent scattering from hydrogens}

Figure 1(a) shows representative constant- $Q$ spectra obtained from the protonated sample PEh at $477 \mathrm{~K}$. As previously commented, they are dominated by the incoherent scattering function of the hydrogens in $\mathrm{PE}, S_{\text {inc }}^{\mathrm{H}}(Q, \omega)$. In the insert of this figure, we can see the $Q$ dependence of the full width at half maximum (FWHM) of the spectra at this temperature. In general, the width of a quasielastic spectrum is related with the inverse of the characteristic time scale of the

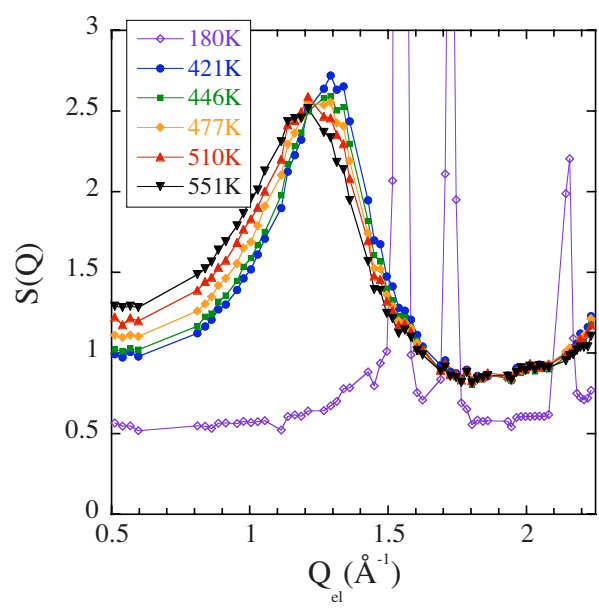

FIG. 2. (Color online) Intensity integrated at constant scattering angle as function of the elastic $Q$ value for the deuterated sample at the temperatures indicated.

motion. With increasing $Q$, the spectra become broader, reflecting thus faster associated characteristic times. This observation, together with the absence of elastic contributions, suggests a diffusivelike behavior of the motions. In Fig. 1(b), the temperature dependence of the spectra shows the expected acceleration of the dynamics (broader curves) when heating the sample.

\section{B. Deuterated sample: Dynamic structure factor}

In contrast to the incoherent spectra, the intensity coherently scattered by the deuterated sample shows a clear modulation with momentum transfer, with a broad maximum centered at $Q \approx 1.3 \AA^{-1}$ in the molten state. As mentioned above, the integral of the coherent scattering function over all frequencies is the static structure factor $S(Q)$. Experimentally, this integral is performed in diffraction experiments, where all neutrons scattered in a given angle are counted irrespective of their energy transfer. We have calculated this function from our constant- $\theta$ spectra for the different temperatures investigated and the results are shown in Fig. 2. The most salient feature of the structure factor at $180 \mathrm{~K}$ in the window explored is the presence of three sharp Bragg peaks. They are signatures of the presence of crystallinity in the sample; in addition, a broad maximum in the region of $Q \approx 1.5 \AA^{-1}$ can be envisaged as well as a tendency to increase in the high- $Q$ range which reveal the weak contribution of a remaining amorphous fraction in the material. Above the melting point, the patterns lack sharp Bragg peaks and show the typical aspect of a liquidlike structure factor. The main broad peak centered in the region of $Q_{\max }$ $\approx 1.25 \AA^{-1}$ slightly shifts toward lower $Q$ values with increasing temperature (from $1.3 \AA^{-1}$ at $421 \mathrm{~K}$ to $1.2 \AA^{-1}$ at $551 \mathrm{~K}$ ). On the other hand, the value of the low- $Q$ plateau of $S(Q)$ increases with temperature. This observation is consistent with an increasing value of the compressibility of the system, but it might also reflect the structural changes that cause the shift and change of the shape of the first peak. The results obtained above $\approx 1.7 \AA^{-1}$ are practically indistinguishable for the different temperatures above the melting. 

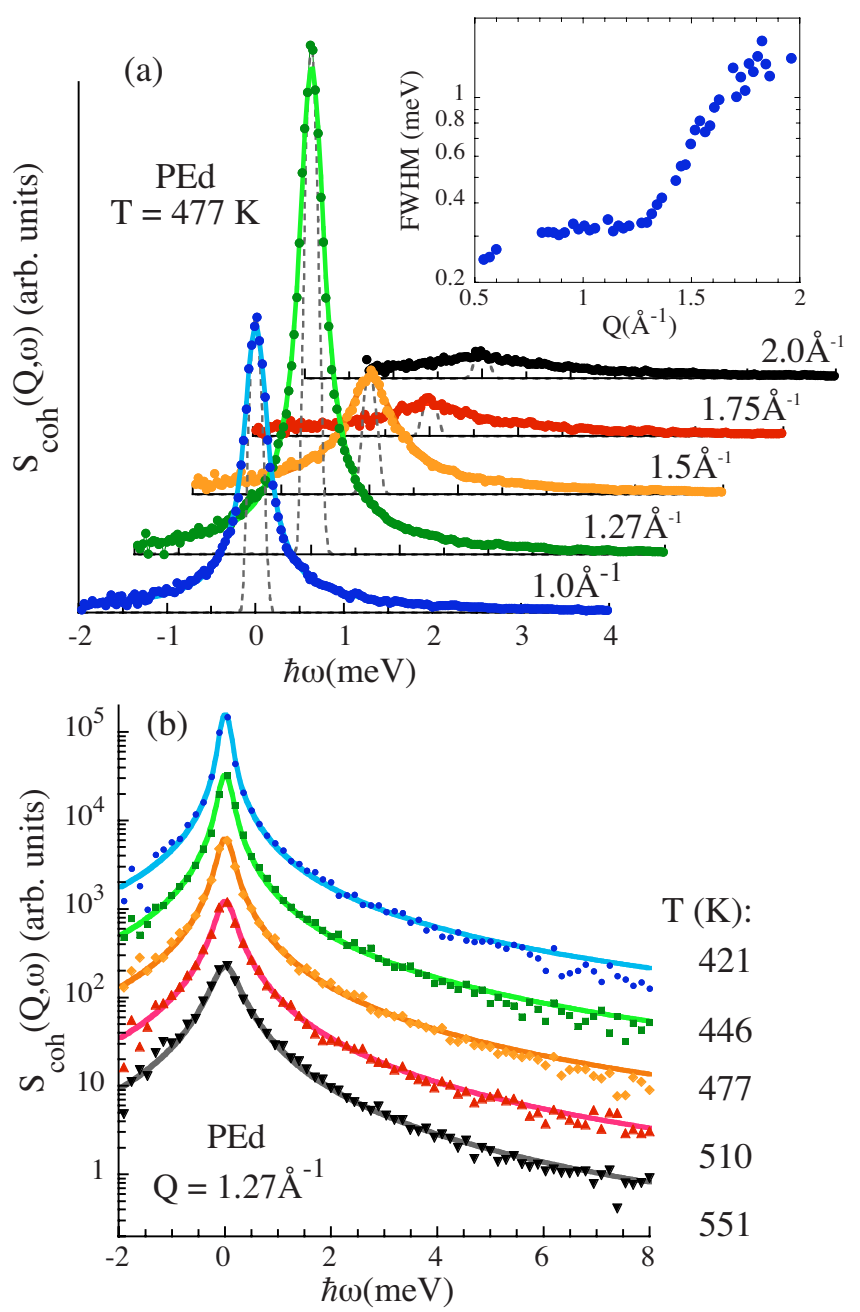

FIG. 3. (Color online) Spectra obtained by interpolation at constant $Q$ for the deuterated sample: (a) at $477 \mathrm{~K}$ and the $Q$ values indicated; (b) at $1.3 \AA^{-1}$ for the different temperatures investigated in a logarithmic representation. In (b), the following factors have been applied for clarity: 16 (421 K), 4 (446 K), 1/4 (510 K), and $1 / 16(551 \mathrm{~K})$. Solid lines through the points are fits with KWW functions. Dotted lines in (a) show the resolution function. Insert in (a) shows the $Q$ dependence of the FWHM of the spectra at $477 \mathrm{~K}$.

Focusing now on the frequency dependence of the dynamic structure factor, Fig. 3(a) displays some spectra at $477 \mathrm{~K}$. For this temperature, the insert shows the $Q$ dependence of the FWHM. An almost constant behavior in the $Q$ range below $Q \approx 1.3 \AA^{-1}$ is followed by a steep increase at higher $Q$ values. On the other hand, Fig. 3(b) shows spectra at $Q=1.3 \AA^{-1}$ (close to the structure factor peak $Q_{\max }$ ) for different temperatures. With increasing temperature, we observe faster dynamics.

\section{DATA ANALYSIS}

We have parametrized our spectra in a phenomenological way by using the versatile functional form of a stretched exponential [Eq. (5)] for the intermediate scattering function. In principle, all three parameters $\left(A, \beta, \tau_{w}\right)$ may depend on both $Q$ and temperature. The Fourier transform of the model

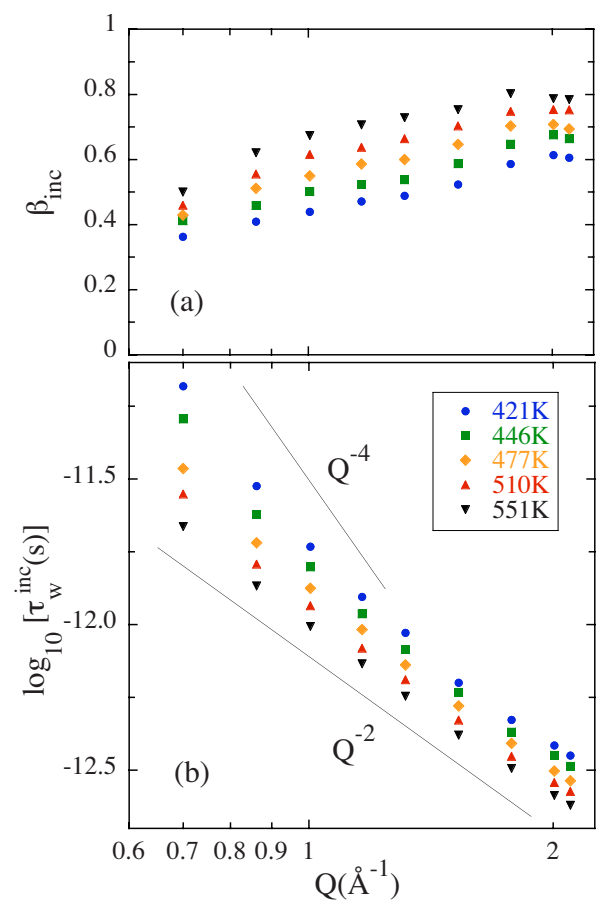

FIG. 4. (Color online) Momentum transfer dependences of (a) the shape parameter and (b) the KWW characteristic time obtained from the KWW descriptions of the incoherent scattering function corresponding to the hydrogens ( $\mathrm{PEh}$ ) at the different temperatures investigated. Solid lines in (b) show $Q^{-2}$ and $Q^{-4}$ dependences.

scattering function in frequency domain was convolved with the instrumental resolution and fitted to the experimental data. As can be appreciated in Figs. 1 and 3, the KWW function describes very well both the incoherent and the coherent spectra. Small deviations can only be envisaged in the low-temperature range investigated and for rather high frequencies above $\approx 6 \mathrm{meV}$ [see Fig. 3(b)].

Figure 4 shows the results obtained for the $\beta$ parameter and characteristic time in the case of the self-motions of hydrogens. The clear increase of the shape parameter with increasing $Q$ and $T$ [Fig. 4(a)] indicates that the intermediate scattering function becomes closer to a single exponential at local length scales and high temperatures. On the other hand, the dispersion of the characteristic time $\tau_{w}^{i n c}$ at high temperatures is close to a $Q^{-2}$ law, while at lower temperatures shows a steeper power law in the low- $Q$ range [Fig. 4(b)]. This implies different temperature behavior of $\tau_{w}^{\text {jnc }}$ for different $Q$ values considered. In the temperature range investigated, the apparent activation energy of this characteristic time continuously increases from $1.4 \mathrm{Kcal} / \mathrm{mol}$ at $Q$ $\gtrsim 1.5 \AA^{-1}$ to $4 \mathrm{Kcal} / \mathrm{mol}$ at $0.7 \AA^{-1}$. Finally, the values deduced for the amplitudes are close to 1 and hardly depend on $Q$ - as expected for the total intensity of incoherent scattering. This implies that the KWW function describes the global decay of the intermediate scattering function-there is no need to include a Lamb-Mössbauer factorlike contribution.

Now turning to the deuterated sample, the results obtained for the three fitting parameters are represented in Fig. 5. Not only the amplitude of the function but also the shape parameter and the characteristic times show oscillations which are 


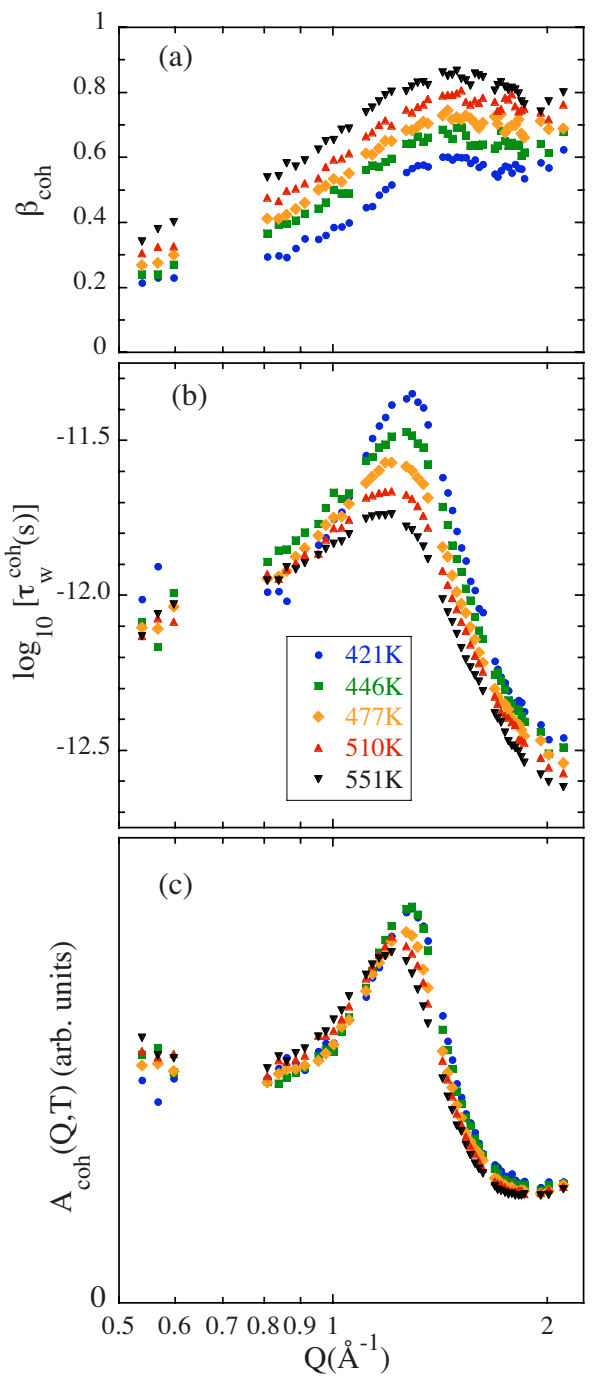

FIG. 5. (Color online) Momentum transfer dependences of (a) the shape parameter, (b) the KWW characteristic time, and (c) the amplitude obtained from the KWW descriptions of the dynamic structure factor $(\mathrm{PEd})$ at the different temperatures investigated.

reminiscent of the maximum of $S(Q)$ at $Q_{\max } \approx 1.3 \AA^{-1}$. This is a clear signature of coherent scattering.

In addition to the analysis in the frequency domain, we have performed the Fourier transformation of the experimentally obtained data into the time domain to obtain the intermediate scattering functions. By division by the Fourier transform of the resolution function, the influence of the instrumental resolution is removed. Figure 6(a) shows as an example the results obtained for the incoherent case, $S_{i n c}^{\mathrm{H}}(Q, t)$, at the intermediate temperature investigated. Here we can appreciate how good are the descriptions of these curves in terms of normalized KWW functions. We also note that, due to the different effective frequency windows accessed by TOF for different $Q$ values, the time range covered varies with momentum transfer. At smaller $Q$ values $\left(\leq 0.9 \AA^{-1}\right)$, the window is restricted to times longer than the picosecond (interpolation beyond $2-5 \mathrm{meV}$ is not possible). On the other hand, it can also be seen that the decay of the correlations is well centered in the TOF window.

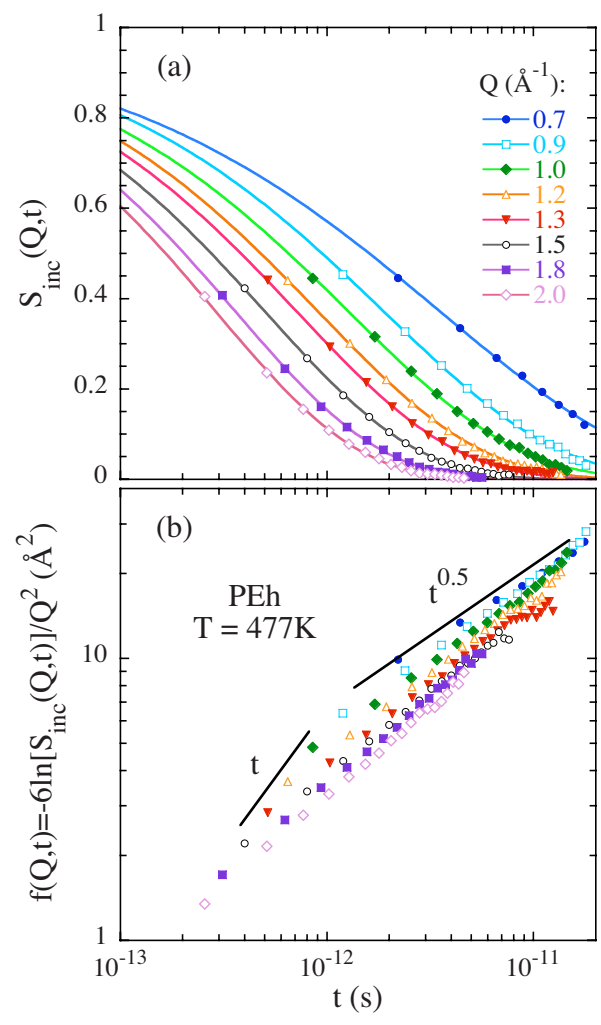

FIG. 6. (Color online) (a) Intermediate incoherent scattering function of PE hydrogens obtained by Fourier transformation of the spectra measured on the PEh sample at $477 \mathrm{~K}$ and the $Q$ values indicated. Solid lines are fits to KWW functions with prefactor fixed to unity. (b) $f(Q, t)$ obtained from the data shown in (a) as defined in Eq. (10). Solid lines are power laws fitting the deduced meansquared displacement (see Fig. 8).

Starting from the correlation function in the time domain $S_{i n c}^{\mathrm{H}}(Q, t)$, we can easily check whether the Gaussian approximation [Eq. (2)] is fulfilled. If so, the function $f(Q, t)$ built as

$$
f(Q, t)=-\frac{6}{Q^{2}} \ln \left[S_{i n c}(Q, t)\right]
$$

is independent of the considered $Q$ value and directly delivers the time-dependent mean-squared displacement of the protons $\left\langle r^{2}(t)\right\rangle$. Figure $6(\mathrm{~b})$ clearly shows that this is not the case for $477 \mathrm{~K}$. Similar results are obtained for the other temperatures investigated. Scrutinizing the $Q$ dependence of $f(Q, t)$, we can extract information on the second-order nonGaussian parameter $\alpha_{2}(t)$ introduced in Eq. (3). To obtain the values of $\left\langle r^{2}(t)\right\rangle$ and $\alpha_{2}(t)$, we have interpolated the function $f(Q, t)$ at different values of the time. Figure 7 shows the results at $477 \mathrm{~K}$ for several times. It turns out that the $Q$ dependence of $f(Q, t)$ cannot be described by considering only the leading correction term, but demands the use of higher-order corrections. Including a further term in the expansion

$$
f(Q, t)=\left\langle r^{2}(t)\right\rangle-\frac{\alpha_{2}(t)\left\langle r^{2}(t)\right\rangle^{2}}{12} Q^{2}+C(t) Q^{4},
$$

the data can be well described. The fit of Eq. (11) delivers the time evolution of $\left\langle r^{2}(t)\right\rangle, \alpha_{2}(t)$, and $C(t)$ shown in Fig. 8 for three of the temperatures investigated. 


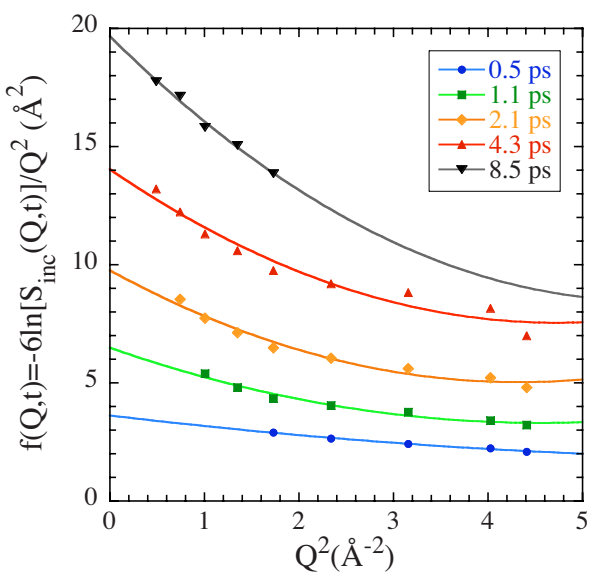

FIG. 7. (Color online) Values of $f(Q, t)$ obtained from interpolation of the result of Eq. (10) for different times at $477 \mathrm{~K}$ versus the variable $Q^{2}$. Lines through the points are fits to Eq. (11).

\section{DISCUSSION}

\section{A. Structure}

The clear temperature dependence of the peak around $1.3 \AA^{-1}$ in $S(Q)$ suggests its interpretation as due to interchain correlations [53]. In fact, the intermolecular character of this first peak has been demonstrated by a careful analysis of the intermolecular hydrogen-hydrogen structure function obtained from neutron-diffraction isotopic substitution experiments [54]. We can then make an estimation of the average distance between neighboring chain segments (belonging to different chains or to the same chain after backfolding) by applying the Bragg approximation $d \approx 2 \pi / Q_{\max }$. This distance increases from $4.8 \AA$ at $421 \mathrm{~K}$ to $5.2 \AA$ at $551 \mathrm{~K}$ (see Table I), presumably due to thermal expansion. These values are in good agreement with that obtained from atomistic MD simulations [17] for the first maximum in the intermolecular mer-mer pair distribution function $(\approx 5.2 \AA$ at $450 \mathrm{~K})$.

On the other hand, the observation of nearly $T$-independent features of $S(Q)$ above $\approx 1.7 \AA^{-1}$ suggests the intramolecular nature of the correlations contributing in this $Q$ range. The presence of the "universal" peak in the $S(Q)$ of polymers located at $Q_{2 \text { max }} \approx 3 \AA^{-1}$ [53] could also be inferred from the incipient increase of the intensity in the high- $Q$ limit of our window. This peak is indeed found in diffraction measurements covering larger momentumtransfer range $[7,54,55]$.

\section{B. Dynamics}

The KWW description of the scattering functions indicates stretching for all $Q$ values and temperatures investigated. Though this stretching is minimized for the structural relaxation $\left[S_{c o h}\left(Q_{\max }, t\right)\right]$, the extrapolation of the $\beta$ value characterizing this function (Table I) to higher temperatures would indicate a value close to $700 \mathrm{~K}$ (i.e., $300 \mathrm{~K}$ above the melting) for the temperature where we would observe a single exponential. Obviously, this range is experimentally inaccessible due to polymer degradation. We now compare the features of the dynamics in the liquid state of PE with

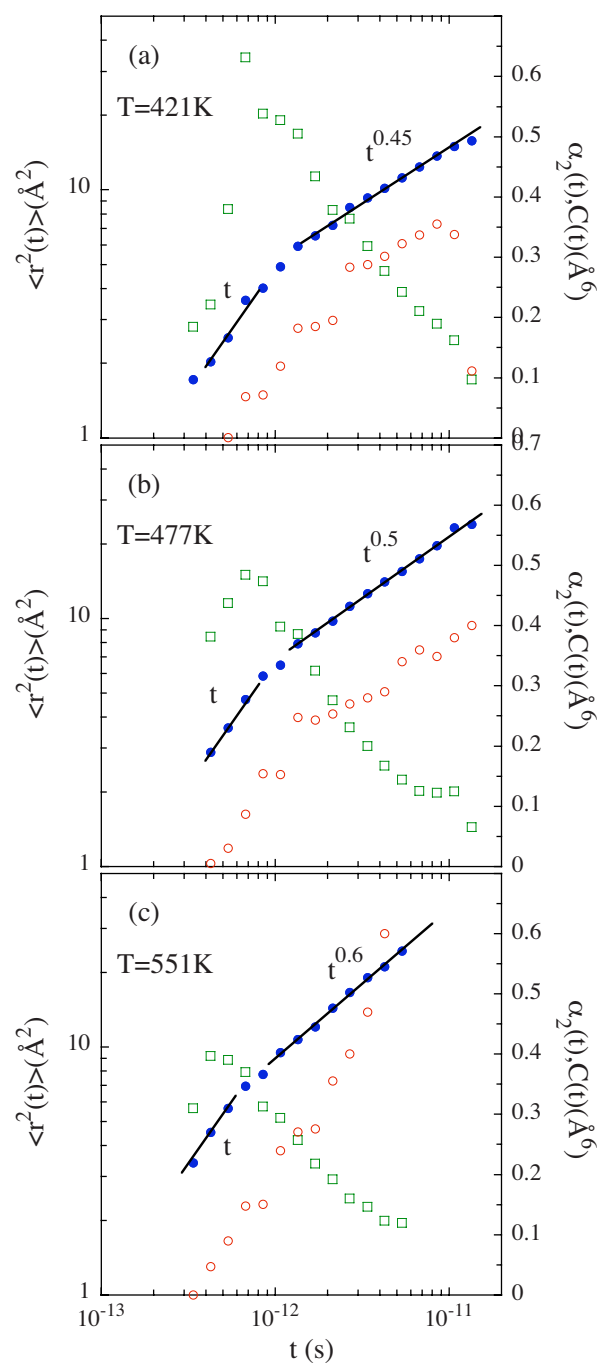

FIG. 8. (Color online) Mean squared displacement (full circles), non-Gaussian parameter (empty squares) and higher-order correction term of the incoherent intermediate scattering function (empty circles) of PE protons at (a) $421 \mathrm{~K}$, (b) $477 \mathrm{~K}$, and (c) $551 \mathrm{~K}$. Solid lines show the linear and sublinear increases of $\left\langle r^{2}(t)\right\rangle$ in the microscopic regime and above, respectively.

those commonly observed in glass-forming polymers in the supercooled regime (Sec. III).

\section{Structural relaxation}

First of all, we consider the features of the structural relaxation as directly followed by $S_{c o h}\left(Q_{\max }, t\right)$. This function is shown in Fig. 9 for the different temperatures investigated. An important point to realize is that $S_{c o h}\left(Q_{\text {max }}, t\right)$ does not display a two-step decay but it evolves in a smooth continuous way in the whole time-window explored [0.5-12 ps] - no signatures of a cage effect can be resolved. However, as pointed out before, the curves show a significant stretching which increases with decreasing temperature.

Figure 10(a) shows the dynamic structure factor in the usual scaling representation applying the shift factors deduced from rheological measurements [56] (reference temperature: $477 \mathrm{~K}$ ). Contrary to the general behavior found for 
TABLE I. Parameters characterizing the structure and dynamics of PE.

\begin{tabular}{|c|c|c|c|c|c|c|c|c|c|}
\hline $\begin{array}{c}T \\
(\mathrm{~K})\end{array}$ & $\begin{array}{c}d \\
(\AA)\end{array}$ & $\beta_{c o h}\left(Q_{\max }\right)$ & $\begin{array}{c}\tau_{w}\left(Q_{\max }\right) \\
(\mathrm{ps})\end{array}$ & $\begin{array}{c}\langle\tau\rangle\left(Q_{\max }\right) \\
\quad(\mathrm{ps})\end{array}$ & $\begin{array}{c}W \ell^{4} \\
\left(\AA^{4} / \mathrm{ps}\right)\end{array}$ & $\begin{array}{c}\ell_{m o n} \\
(\AA)\end{array}$ & $\begin{array}{l}\tau_{m o n} \\
(\mathrm{ps})\end{array}$ & $\begin{array}{l}\ell_{\text {seg }} \\
(\AA)\end{array}$ & $\begin{array}{l}\tau_{\text {seg }} \\
(\mathrm{ps})\end{array}$ \\
\hline 421 & 4.8 & 0.57 & 4.5 & 7.3 & 16.0 & 5.9 & 7.9 & 13.2 & 197 \\
\hline 446 & 4.9 & 0.64 & 3.3 & 4.5 & 27.1 & 5.9 & 4.4 & 13.1 & 109 \\
\hline 477 & 5.0 & 0.68 & 2.7 & 3.4 & 42.9 & 5.8 & 2.6 & 12.8 & 64.3 \\
\hline 510 & 5.1 & 0.70 & 2.1 & 2.7 & 60.6 & 5.6 & 1.7 & 12.6 & 42.2 \\
\hline 551 & 5.2 & 0.80 & 1.8 & 2.1 & 82.2 & 5.5 & 1.1 & 12.3 & 28.4 \\
\hline
\end{tabular}

the $\alpha$ relaxation in polymers in the NSE time window, the data do not superimpose in a single master curve. In Fig. 10(b), arbitrary shift factors have been applied to the time scale to achieve the best superposition of the decays; this is only possible however in the long-time tails of the data. Thus, the time-temperature superposition principle which is generally observed in supercooled polymers for the $\alpha$ relaxation still works in PE at long times (above $\approx 2-3 \mathrm{ps}$ ) and fails in the microscopic region. The applied shift factors (shown as circles in the insert of the figure) can be rather well described with an Arrhenius-like dependence with an activation energy of $5 \mathrm{Kcal} / \mathrm{mol}$, significantly lower than the apparent activation energy shown by the extrapolation of the rheological data in this temperature range $(\approx 8 \mathrm{Kcal} / \mathrm{mol})$. Seemingly, above the melting, the cooperativity effects giving rise to the Vogel-Fulcher dependence observed at lower temperatures are much less significant. We note that the observed activation energy approaches that in the isolated chain $(3.25 \mathrm{Kcal} / \mathrm{mol})$ as obtained in the simulations by Boyd et al. [15].

Due to the change in spectral shape when considering different temperatures, the average relaxation time $\langle\tau\rangle$ (first moment of the equivalent distribution of relaxation times) might be a more representative parameter to discuss the results. In the case of KWW functions, $\langle\tau\rangle$ can be obtained as

$$
\langle\tau\rangle=\Gamma\left(\frac{1}{\beta}\right) \frac{\tau_{w}}{\beta} .
$$

In fact, we note that the temperature dependence of $\left\langle\tau_{c o h}\left(Q_{\max }, T\right)\right\rangle$ is very close to that of the shift factors ob-

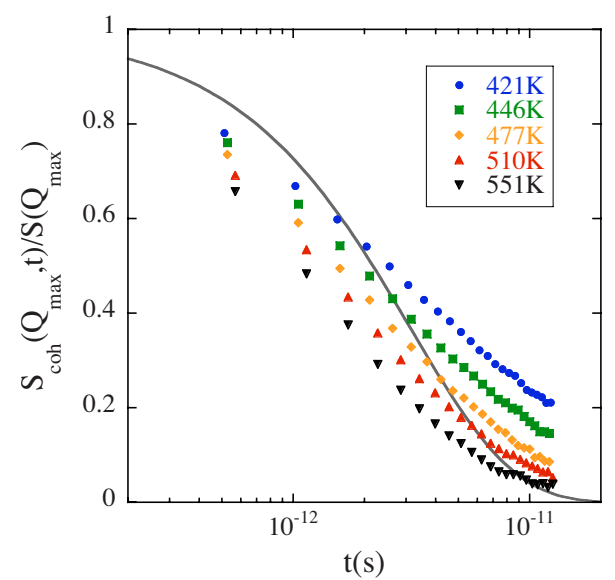

FIG. 9. (Color online) Normalized dynamic structure factor at its first maximum for the different temperatures investigated. The line shows a single exponential decay. tained from the superposition of $S_{c o h}\left(Q_{\max }, t\right)$, while $\tau_{w}^{c o h}\left(Q_{\max }, T\right)$ shows a weaker dependence (apparent activation energy: $3.4 \mathrm{Kcal} / \mathrm{mol}$-almost identical to that of the isolated chain). In the following, we will therefore consider $\left\langle\tau_{\text {coh }}\left(Q_{\text {max }}, T\right)\right\rangle$ as the representative value for the structural relaxation time $\tau_{\alpha}$ (see Table I).

\section{Hydrogen motions}

The self-motions of hydrogens also show a different behavior than that expected at lower temperatures. Salient features are the $Q$ - and $T$-dependent spectral shapes of the incoherent scattering function and the close to $Q^{-2}$ law observed for the characteristic time at the highest tempera-

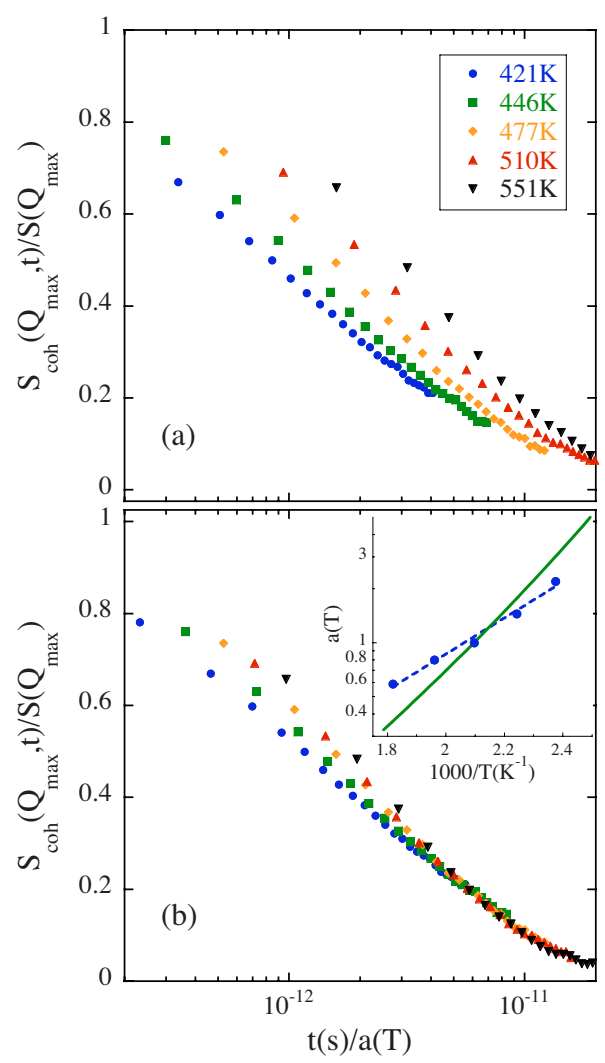

FIG. 10. (Color online) Normalized dynamic structure factor at its first maximum represented as function of scaled time variable using (a) rheological shift factors [56] and (b) free shift factors. The values of the shift factors used are shown in the insert of (b) (rheological: solid line; free: symbols; Arrhenius fit of free shift factors: dashed line). 
ture (Fig. 4). They do not correspond either to the standard behavior in the $\alpha$-relaxation regime $\left(\beta \approx 0.5, \tau \propto Q^{-2 / \beta}\right)$, or a Rouse-like motion $\left(\beta=0.5, \tau \propto Q^{-4}\right)$, or to a pure diffusive behavior $\left(\beta=1, \tau \propto Q^{-2}\right)$; moreover, due to the $Q$ dependence of the spectral shape, it is difficult to elucidate, for example, whether or where the Gaussian approximation is fulfilled. Finally, we note the nearly constant amplitude factors obtained from the KWW descriptions of the scattering functions.

The interpretation of these observations is best realized considering the results in the time domain. In a similar way as $S_{\text {coh }}\left(Q_{\text {max }}, t\right)$, the intermediate incoherent scattering function lacks a two-step decay in the accessed window [see Fig. 6(a)]. The mean-squared displacement of the hydrogens displays a clear direct crossover from a linear increase at microscopic times below $\approx 1$ ps toward a sublinear regime at longer times [Fig. 8]. This indicates that the dynamics is so fast that the $\mathrm{H}$ atoms do not feel any cage effect from neighboring chains. The value of the exponent in the sublinear regime tends to slightly increase with increasing temperature, from 0.45 at $421 \mathrm{~K}$ to 0.6 at $551 \mathrm{~K}$. On the other hand, the non-Gaussian parameter shows only one maximum located in the microscopic region. With increasing temperature, the value of $\alpha_{2}$ decreases and the position of the maximum slightly shifts to faster times $(0.9$ ps for $421 \mathrm{~K}, 0.7 \mathrm{ps}$ for $477 \mathrm{~K}$, and $0.4 \mathrm{ps}$ for $551 \mathrm{~K}$ ). There is no clear signature of the strongly temperature-dependent second peak of $\alpha_{2}(t)$ which usually appears in the crossover region from microscopic to subdiffusive behavior attributed to the local events leading to the decaging process [34,38]. In Fig. 11, we compare the evolution of the mean squared displacement of $\mathrm{H}$ atoms to the decay of the intermolecular correlations at the two extreme temperatures investigated. The structural relaxation time $\tau_{\alpha}$ is located very close to the time where the direct crossover takes place. This implies that, if there would be a cage effect, the decaging process would take place already in the microscopic regime. For comparison, Fig. 11 also shows the time $\tau_{d}$ when the displacement of a $\mathrm{H}$ atom reaches the intermolecular distance that is about 4 times longer than the structural relaxation time $\tau_{\alpha}$.

Thus, in the high-temperature range explored for PE, we can say that the decaging process has practically merged with the microscopic dynamics. Consequently, in the $Q, t$, and $T$ ranges investigated, $S_{i n c}(Q, t)$ decays in a single step. The drastic change from linear to sublinear increase of the mean-squared displacement in the dynamic window explored translates into a strongly $Q$-dependent shape parameter of the scattering function [Fig. 4(a)]. At large $Q$ values (small length scales), the dynamics is dominated by the microscopic motions (vibrations, librations) [18], which are not very much affected by correlation effects (and thereby tend to a Debye-like functional form). In fact, at lower temperatures, other polymers show Debye-like relaxation in the microscopic regime. In those cases, we note that the Debye description of the intermediate scattering functions was limited to the very short regime, below $\approx 1$ ps. Here, the whole decay is considered and at the latest times, the functions might be influenced by correlated processes or other processes with slightly different time scales. At small $Q$ values (large length scales), the diffusivelike processes taking place

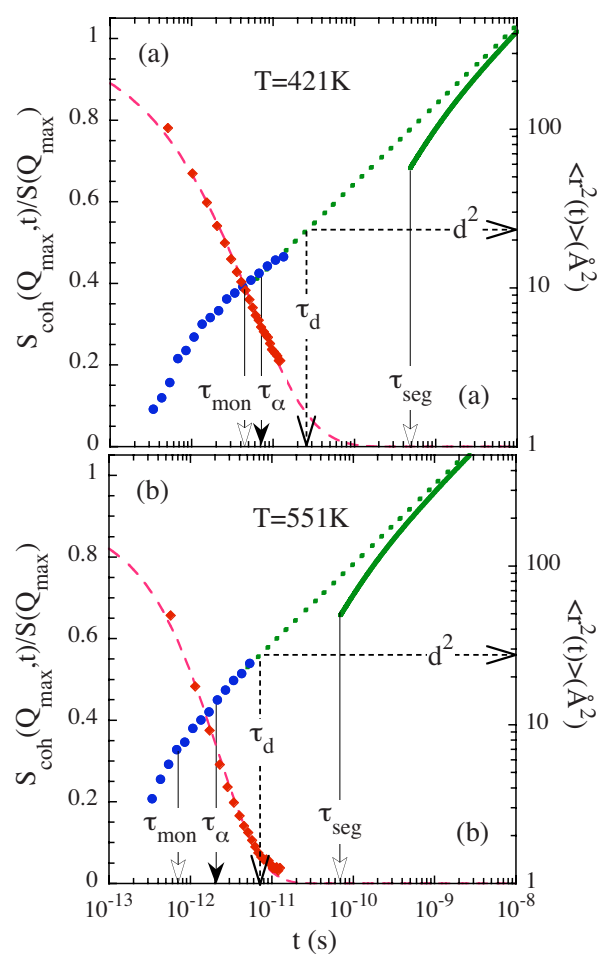

FIG. 11. (Color online) Normalized dynamic structure factor (full diamonds) and mean-squared displacement of PE protons (full circles) at (a) $421 \mathrm{~K}$ and (b) $551 \mathrm{~K}$. Dashed lines through the dynamic structure factor are KWW fits. Solid lines are the Rouse predictions $\left\langle r^{2}(t)\right\rangle_{\text {Rouse }}$ considering 1 bead $=10 \mathrm{CH}_{2}$ units and the dotted lines the asymptotic laws $\left\langle r^{2}(t)\right\rangle_{\text {Rouse }}^{p \ll N-1}$. Arrows mark the position of the structural relaxation time $\tau_{\alpha}$, the monomeric time $\tau_{\text {mon }}$, and the segmental time $\tau_{\text {seg }}$ in the Rouse model (see text) and the time $\tau_{d}$ when the proton covers the interchain distance $d$.

via torsional transitions $[15,18]$ dominate. Due to chain connectivity, the diffusion is anomalous, leading to stretched decays of the correlation function.

It is worth noting that an observation of mixed decaging and microscopic dynamics has also been reported for medium-chain $n$ alkanes in a recent work [57]. Using resolution resolved quasielastic TOF combined with MD simulations, different dominant motions of the molecule were disentangled: tumbling motions of the $\mathrm{H}$ atoms around and with the $\mathrm{C}$ atoms, small chain deformations and partial or total exchange of the place of the molecule with its neighbors, leading finally to Fickian long-range diffusion. Probably all the local motions identified for $n$ alkanes are also active in the long PE chains; the main qualitative difference arises naturally from the chain connectivity that for long times and large length scales causes Rouse-like dynamics in PE against the "normal" diffusion in the oligomers. This leads to stretched functional forms for PE while $n$ alkanes exhibit Lorentzian diffusive components.

In Fig. 8, we can see that the non-Gaussian parameter $\alpha_{2}(t)$ takes significant values $\left[\alpha_{2}(t) \geqslant 0.2 \ldots 0.3[34,39]\right]$ at times shorter than $t_{c}\left[t_{c}(421 \mathrm{~K}) \approx 5 \mathrm{ps}, t_{c}(551 \mathrm{~K})\right.$ $\approx 1.5 \mathrm{ps}$. Inspecting Fig. $4(\mathrm{~b})$, we realize that $\tau_{w}^{i n c}(Q, T)$ is smaller or of the order of $t_{c}$ for $Q \gtrsim 0.8 \AA^{-1}$. This implies that for $Q \gtrsim 0.8 \AA^{-1}$, the incoherent scattering function is 
strongly non-Gaussian, an observation which is consistent with the results of Fig. 6(b).

The incoherent scattering function of PE hydrogens has been investigated in a number of previous works (see, e.g., $[6-8,10-12,16,18,19])$. Some of those studies were focused on the lower temperature region, where the sample is semicrystalline $[6,8,10]$. Above the melting point, the analysis of the data in terms of KWW functions delivered very similar results ( $\beta$ values and dispersions of the characteristic times) to those here reported. The analysis in the time domain was addressed in Refs. [16,18] where the function $f(Q, t)$ was obtained in a similar way from TOF data. Deviations from Gaussian behavior were also reported though they were not quantified. In fact, the only experimental information about the mean-squared displacement and the $\alpha_{2}$ parameter was provided in Ref. [11] in the solid state (amorphous and crystalline) from elastic measurements. They reported values for $\alpha_{2}$ in the range $0.2 \ldots 0.3$.

The question of the Gaussian behavior can be much more easily addressed with computer simulations at hand, which allow direct insight in the correlations in real space (and can avoid crystallization). The results obtained at $504 \mathrm{~K}$ from a united-atom PE model by Arialdi et al. [20] showed $\alpha_{2}(t$ $\geq 1 \mathrm{ps})<0.1$ and a tendency to increase toward shorter times, very much compatible with our observation. Also comparable are the results obtained by Roe [14] in a PE united-atom model at lower temperatures. Using an explicit atom PE model, Smith et al. [16,18] also found deviations from Gaussian behavior that were attributed to the onset of torsional transitions. Regarding other polymers at equivalently high temperatures, recent MD simulations on polyethylene oxide [50] show a very similar behavior of the nonGaussian parameter to that here reported for PE; also consistently, the second peak of $\alpha_{2}(t)$ in polyisoprene [34] was shown to disappear (merged with the microscopic one) at very high temperatures.

Finally, we comment on the NSE work on protonated PE reported in Ref. [12]. There, the intermediate scattering function was measured at $509 \mathrm{~K}$ and times above $10 \mathrm{ps}$. The function $f(Q, t)$ [Eq. (10)] corresponding to two $Q$ values superimposed well, suggesting Gaussian behavior in the explored time region. Those results are consistent with ours, since for $t>10 \mathrm{ps}$, we extrapolate a very small value for $\alpha_{2}$; moreover, the values reported for the mean-squared displacement agree in both cases, $\left\langle r^{2}(t \approx 10 \mathrm{ps})\right\rangle \approx 25 \AA^{2}$. In that work, the motions observed were interpreted in terms of the Rouse model up to $1 \mathrm{~ns}$, where the influence of the topological constraints becomes apparent. In the following, we discuss how our results compare to the Rouse prediction.

\section{Connection with Rouse dynamics}

Consistently with the experiments on incoherent scattering [12], NSE results on the single chain dynamic structure factor of PE fully agree with the Rouse model where entanglement effects are not important [58]. In that work, the values of the Rouse variable $W \ell^{4}$ were reported at temperatures in the range here investigated. In Table I, we show the $W \ell^{4}$ values interpolated according to the best Vogel-Fulcher fit of those results: $W \ell^{4}\left[\AA^{4} / \mathrm{ps}\right]$
$=4.15 \times 10^{10} \exp [-417.67 /(\mathrm{T}[\mathrm{K}]-292.77)][59]$. The meansquared displacements deduced from the Rouse model [Eq. (7)] with such values of $W \ell^{4}$ have been included as solid lines in Fig. 11. In this calculation, we have considered the statistical segment in the definition of the bead. Neutron scattering measurements on the chain conformation report a segment size of about $10 \mathrm{CH}_{2}$ units [30,60,61]; MD simulations [62] found a similar result $\left(9 \mathrm{CH}_{2}\right)$. We have chosen 1 bead $=10 \mathrm{CH}_{2}$ units. The corresponding temperaturedependent sizes $\ell_{\text {seg }}$ have been obtained from Ref. [30] and are displayed in Table I. In principle, the validity of the Rouse model is limited to length scales larger than $\ell_{\text {seg. }}$. This table also shows the values of the segmental time $\tau_{\text {seg }}$ (characteristic time of the highest mode number $p$ in the Rouse model) that is about 2 orders of magnitude slower than the structural relaxation time $\tau_{\alpha}$. Figure 11 shows that, in order to match the experimental results with the Rouse prediction, we would have to assume some change in the slope of $\left\langle r^{2}\right\rangle$ in the gap between the limit of our experimental window and $\tau_{\text {seg }}$. However, the extrapolation toward short times of the asymptotic low- $p$ results for the Rouse mean-squared displacement [Eq. (8)] matches the experimental results astonishingly well. If we attribute the sublinear regime to Rouselike motion as suggested by this coincidence, we should assume a much smaller bead size than the statistical segment. For the case 1 bead=1 $\mathrm{CH}_{2}$ monomer, the shortest time in the Rouse motion $\tau_{\text {mon }}$ would be even faster than the structural relaxation time and the Rouse prediction would describe rather well the experimental data above $\tau_{\text {mon }}$ (see Fig. 11 and Table I). Thus, from the TOF results, it could be concluded that the Rouse model effectively describes the whole dynamics after the microscopic regime, even if in principle it should not be applicable in this regime.

Analysis of the individual Rouse modes can be made from MD-simulation results. In fact, several authors report deviations from the Rouse predictions for PE $[17,18]$. Smith et al. $[16,18]$ attributed the observed deviations primarily to the non-Gaussian self-displacements resulting from intermolecular correlations. We thus face a somehow paradoxical situation: the Rouse model indeed fails at short times and small length scales, but, within the uncertainties, the experimental observation would be compatible with it even where it cannot be applicable. A similar situation has been found in the case of polyethylene oxide [50].

\section{Collective versus self-motions}

The phenomenological KWW description of the scattering functions requires $Q$ - and $T$-dependent shape parameters that also depend on the correlation function considered. Therefore, for a direct comparison of the coherent and incoherent characteristic times, we consider the average times as defined in Eq. (12). They are displayed in Fig. 12 for three temperatures. The reminiscence of $S(Q)$ in the coherent average times is clear.

In the case of simple monoatomic liquids, the coherent scattering function can easily be built starting from the incoherent one. There, the coherent characteristic time shows the so-called de Gennes narrowing [5] 


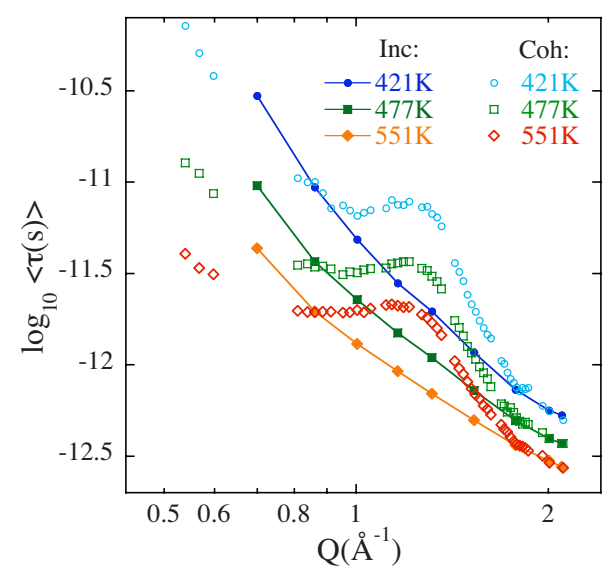

FIG. 12. (Color online) Comparison between the average times obtained for the incoherent (full symbols connected with solid lines) and coherent (empty symbols) scattering functions at three temperatures.

$$
\tau_{\text {coh }}(Q)=S(Q) \tau_{\text {inc }}(Q)
$$

We may check how well does it work for the characteristic times of PE. Figure 13(b) shows the ratio between the coherent and incoherent average times. In the same figure, the structure factor at the two extreme temperatures has been included [63]. We find clear discrepancies, especially below

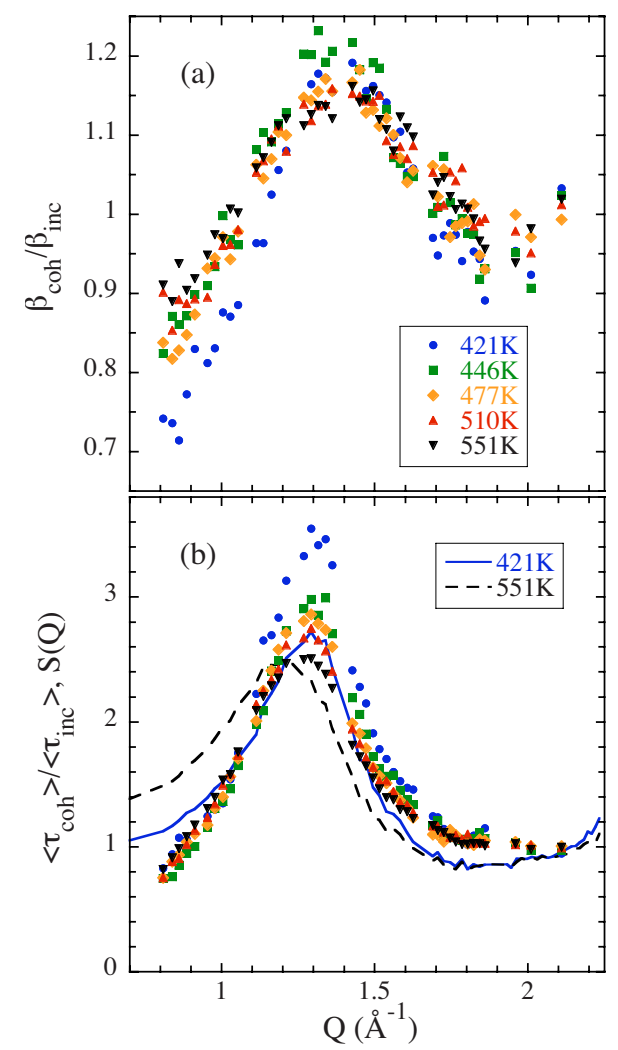

FIG. 13. (Color online) Momentum transfer dependence of the ratio between (a) the shape parameters and (b) the average times corresponding to coherent and incoherent scatterings. Lines show the structure factor at the two extreme temperatures. the first maximum of $S(Q)$. It is also noteworthy that for the lowest temperature investigated, the behavior in the peak region is more marked, leading there to even stronger deviations from Eq. (13).

We note that the measured incoherent scattering function corresponds to a subsystem of the atoms in PE (only the hydrogens) and does not necessarily coincide with the self part of the coherent scattering function, i.e., which includes also the carbons. Unfortunately, this function is not accessible experimentally (the incoherent cross section of $\mathrm{C}$ is zero). We could expect that hydrogens move faster than carbons, leading to larger values of the ratio between the experimental times than considering the right self-correlation function. This could explain the differences above $Q_{\max }$. However, at lower $Q$ s, such an argument does not reconcile the comparison. There, we also note the markedly stretched behavior of the dynamic structure factor [see Fig. 5(a)] and the high values obtained for the amplitudes (larger than 1 for the normalized functions). This implies that the KWW description in this regime has to be considered as a mere effective description of the data in the experimentally accessible dynamic window. At shorter times, it must necessarily fail. We also note that the failure of de Gennes prediction at low $Q$ values cannot be attributed to the incoherent contribution of deuterons to the spectra. This contribution should be slower than the collective dynamics. Considering now the spectral shapes, the ratio between the shape parameters $\beta$ shows a significant $S(Q)$-like modulation in [Fig. 13(a)], being at $Q \gtrsim 1.5 \AA^{-1}$ rather close to 1 .

As mentioned above (Sec. III), at lower temperatures, the first fast regime has been found to follow a Debye-like behavior for both coherent and incoherent scatterings. The coherent characteristic times deduced for polybutadiene $(\mathrm{PB})$ in the fast dynamics regime followed rather well the $Q$ dependence of a simple de Gennes narrowinglike relationship with the incoherent times deduced from the protonated samples [27]. In a similar way, within the experimental limitations, such a relationship seems to be also fulfilled for poly(vinyl chloride) $[28,64,65]$. The situation is more complicated when the second slow decay of the dynamic structure factor is considered. In the case of $\mathrm{PB}$, its reminiscence of $S(Q)$ could be attributed to the $\beta$ process [66]. In polyisobutylene (PIB), this decay shows a $S(Q)$ modulation in the amplitude, but a $Q$-independent $\beta$ parameter. Close to the peak, the coherent characteristic times could be approximately described with a Sköld-like ansatz [67] that might be more appropriated for stretched exponentials representing anomalous diffusion [68]

$$
\tau_{\text {coh }}(Q)=S(Q)^{1 / \beta} \tau_{\text {inc }}(Q) .
$$

This expression produces a sharper modulation with $S(Q)$ than that expected in the case of simple liquids $(\beta=1)$. However, this description fails again at low $Q$ in PIB [35]. In poly(vinyl acetate) (PVAc), the spectral shape is approximately independent of $Q$; both de Gennes and Sköld approaches fail in accounting for the observed narrowing. The complementary MD simulations in that work on PVAc and in Ref. [42] on poly(methyl methacrylate) reveal the intriguing complexity of the correlations contributing at the first peak in polymers containing side groups, ruling out the possibility of 
applying such simple approaches to collective dynamics in such systems.

In $\mathrm{PE}$, at high $Q$ values, we observe a rather similar behavior to that reported for the microscopic dynamics at lower temperatures. The only difference is the stretching that we have rationalized above. At lower $Q$ values, the correlation functions in PE decay to a large extent through diffusivelike motions that are strongly influenced by chain connectivity leading not only to stretching, but also to severe deviations from the $Q$ dependence expected in a simple liquid. Using Eq. (14) does not improve the description of $\left\langle\tau_{\text {coh }}\right\rangle /\left\langle\tau_{\text {inc }}\right\rangle$. However, we note that the sharper feature observed at $421 \mathrm{~K}$ (Fig. 13) could hint the incipient emergence of the signatures found at lower temperatures when the two-step relaxation is well developed.

\section{CONCLUSIONS}

We have characterized the coherent and incoherent scattering functions measured by time-of-flight techniques on polyethylene above the melting point. From the results, we conclude that:

(i) In the time-range accessed, the intermediate scattering functions display a single-step decay. No signatures of cage effect are visible.

(ii) The structural relaxation as observed at the intermolecular structure factor peak shows a weaker temperature dependence than that extrapolated from macroscopic mechanical measurements and approaching to that obtained for an isolated chain [15], indicating a low degree of interchain cooperativity.

(iii) There is an apparent direct crossover from the microscopic regime to a Rouse-like regime (though MD simulations show evidences of deviations from Rouse dynamics).

(iv) In the high $Q$ range-local scales-the scattering functions decay mainly through fast processes (vibrations, librations, tumbling of $\mathrm{CH}_{2}$ groups, etc.) [18,57]. There, correlations are less apparent and the stretching could be due to the heterogeneity in the time scales of these local motions. At larger length scales (intermolecular and in the so-called intermediate-length-scales region), the intermediate scattering functions decay to a rather significant extent via torsional transitions $[15,18]$ that determine the chain dynamics. There, chain connectivity plays a major role in the stretching observed for the scattering functions.

(v) A simple de Gennes-like narrowing could reasonably describe the behavior at $Q$ values above the structure factor peak (apart from the remaining stretching). However, at lower $Q$ values, the relation between coherent and incoherent scattering functions is not reproduced by any existing approach. The markedly stretched behavior of the dynamic structure factor at intermediate-length scales is particularly intriguing.

Predicting the behavior of the dynamic structure factor in the intermediate-length scales region is by no means trivial [35]. This work may motivate further theoretical or simulation efforts to understand the dynamics of polymers in this almost unexplored region. The new experimental results here presented provide essential information to validate MD simulations and/or or check theoretical approaches in this direction.

\section{ACKNOWLEDGMENTS}

We thank R. Kahn for experimental support at MIBEMOL and Laboratoire Léon Brillouin, CEA-Saclay (France) for neutron facilities. This research project has been supported by the European Commission NoE SoftComp Contract No. NMP3-CT-2004-502235, the "Donostia International Physics Center," the Projects No. MAT2007-63681 and No. IT-436-07 (GV), and the Spanish Ministerio de Educacion y Ciencia (Grant No. CSD2006-53).
[1] W. Götze, in Liquids, Freezing, Glass Transition, edited by J. P. Hansen, D. Levesque, and J. Zinn-Justin (North-Holland, Amsterdam, 1991), p. 287.

[2] K. L. Ngai, Comments Solid State Phys. 9, 121 (1979).

[3] W. Götze, J. Phys.: Condens. Matter 11, A1 (1999).

[4] A. Brodin and E. A. Rössler, J. Phys.: Condens. Matter 18, 8481 (2006).

[5] P. G. de Gennes, Physica (Amsterdam) 25, 825 (1959).

[6] T. Peterlin-Neumaier and T. Springer, J. Polym. Sci., Polym. Phys. Ed. 14, 1351 (1976).

[7] U. Buchenau, M. Monkenbusch, M. Stamm, C. F. Majkrzak, and N. Nücker, in Polymer Motion in Dense Systems, edited by D. Richter and T. Springer, Springer Proceedings in Physics Vol. 29 (Springer, New York, 1988), p. 138.

[8] G. Hohlweg, B. Holzer, W. Petry, G. Strobl, and B. Stühn, Macromolecules 25, 6248 (1992).

[9] B. Holzer, G. Strobl, B. Stühn, and N. H. Andersen, Colloid Polym. Sci. 272, 1396 (1994).

[10] T. Kanaya, T. Kawaguchi, and K. Kaji, Macromolecules 32,
1672 (1999).

[11] T. Kanaya, U. Buchenau, S. Koizumi, I. Tsukushi, and K. Kaji, Phys. Rev. B 61, R6451 (2000).

[12] A. Wischnewski, M. Monkenbusch, L. Willner, D. Richter, and G. Kali, Phys. Rev. Lett. 90, 058302 (2003).

[13] H. Takeuchi and R.-J. Roe, J. Chem. Phys. 94, 7458 (1991).

[14] R.-J. Roe, J. Chem. Phys. 100, 1610 (1994).

[15] R. H. Boyd, R. H. Gee, J. Han, and Y. Jin, J. Chem. Phys. 101, 788 (1994).

[16] G. D. Smith, W. Paul, D. Y. Yoon, A. Zirkel, J. Hendricks, D. Richter, and H. Schober, J. Chem. Phys. 107, 4751 (1997).

[17] V. A. Harmandaris, V. G. Mavrantzas, and D. N. Theodorou, Macromolecules 31, 7934 (1998).

[18] G. D. Smith, W. Paul, M. Monkenbusch, and D. Richter, Chem. Phys. 261, 61 (2000).

[19] G. Arialdi, K. Karatasos, J.-P. Ryckaert, V. Arrighi, F. Saggio, A. Triolo, A. Desmedt, J. Pieper, and R. E. Lechner, Macromolecules 36, 8864 (2003).

[20] G. Arialdi, J.-P. Ryckaert, and D. N. Theodorou, Chem. Phys. 
292, 371 (2003).

[21] T. Springer, Quasielastic Neutron Scattering for the Investigation of Diffusive Motions in Solids, Liquids, Springer Tracts in Modern Physics (Springer-Verlag, Berlin, 1972), Vol. 64.

[22] S. W. Lovesey, Theory of Neutron Scattering from Condensed Matter (Clarendon Press, Oxford, 1984).

[23] G. L. Squires, Introduction to the Theory of Thermal Neutron Scattering (Dover Publication Inc., New York, 1996).

[24] A. Rahman, K. S. Singwi, and A. Sjölander, Phys. Rev. 126, 986 (1962).

[25] R. Zorn, Phys. Rev. B 55, 6249 (1997).

[26] J. Colmenero, A. Arbe, and A. Alegría, Phys. Rev. Lett. 71, 2603 (1993).

[27] R. Zorn, A. Arbe, J. Colmenero, B. Frick, D. Richter, and U. Buchenau, Phys. Rev. E 52, 781 (1995).

[28] J. Colmenero, A. Arbe, G. Coddens, B. Frick, C. Mijangos, and H. Reinecke, Phys. Rev. Lett. 78, 1928 (1997).

[29] J. Colmenero and A. Arbe, Phys. Rev. B 57, 13508 (1998).

[30] D. Richter, M. Monkenbusch, A. Arbe, and J. Colmenero, Neutron Spin Echo in Polymer Systems, Advanced Polymer Science Vol. 174 (Springer-Verlag, Berlin, 2005).

[31] J. Colmenero, A. Alegría, A. Arbe, and B. Frick, Phys. Rev. Lett. 69, 478 (1992).

[32] A. Arbe, J. Colmenero, M. Monkenbusch, and D. Richter, Phys. Rev. Lett. 81, 590 (1998).

[33] J. Colmenero, A. Arbe, A. Alegría, M. Monkenbusch, and D. Richter, J. Phys.: Condens. Matter 11, A363 (1999).

[34] J. Colmenero, F. Alvarez, and A. Arbe, Phys. Rev. E 65, 041804 (2002).

[35] B. Farago, A. Arbe, J. Colmenero, R. Faust, U. Buchenau, and D. Richter, Phys. Rev. E 65, 051803 (2002).

[36] A. Arbe, J. Colmenero, F. Alvarez, M. Monkenbusch, D. Richter, B. Farago, and B. Frick, Phys. Rev. Lett. 89, 245701 (2002).

[37] A. Triolo, R. E. Lechner, A. Desmedt, M. T. F. Telling, and V. Arrighi, Macromolecules 35, 7039 (2002).

[38] A. Arbe, J. Colmenero, F. Alvarez, M. Monkenbusch, D. Richter, B. Farago, and B. Frick, Phys. Rev. E 67, 051802 (2003).

[39] A. Narros, F. Alvarez, A. Arbe, J. Colmenero, D. Richter, and B. Farago, J. Chem. Phys. 121, 3282 (2004).

[40] M. Tyagi, A. Alegría, and J. Colmenero, J. Chem. Phys. 122, 244909 (2005).

[41] R. Pérez Aparicio, A. Arbe, J. Colmenero, B. Frick, L. Willner, D. Richter, and L. J. Fetters, Macromolecules 39, 1060 (2006).

[42] A.-C. Genix, A. Arbe, F. Alvarez, J. Colmenero, B. Farago, A. Wischnewski, and D. Richter, Macromolecules 39, 6260 (2006).

[43] M. Tyagi, A. Arbe, F. Alvarez, J. Colmenero, and M. A. González, J. Chem. Phys. 129, 224903 (2008).

[44] D. Richter, M. Monkenbusch, L. Willner, A. Arbe, J. Colmenero, and B. Farago, Europhys. Lett. 66, 239 (2004).
[45] P. E. Rouse Jr., J. Chem. Phys. 21, 1272 (1953).

[46] M. Doi and S. F. Edwards, The Theory of Polymer Dynamics (Oxford University Press, Oxford, England, 1986).

[47] A. Arbe, Physica B 350, 178 (2004).

[48] A.-C. Genix, A. Arbe, F. Alvarez, J. Colmenero, L. Willner, and D. Richter, Phys. Rev. E 72, 031808 (2005).

[49] M. Tyagi, A. Arbe, J. Colmenero, B. Frick, and J. R. Stewart, Macromolecules 39, 3007 (2006).

[50] M. Brodeck, F. Alvarez, A. Arbe, F. Juranyi, T. Unruh, O. Holderer, J. Colmenero, and D. Richter, J. Chem. Phys. 130, 094908 (2009).

[51] D. Richter, M. Monkenbusch, J. Allgaier, A. Arbe, J. Colmenero, B. Farago, Y. Cheol Bae, and R. Faust, J. Chem. Phys. 111, 6107 (1999).

[52] A. Arbe, M. Monkenbusch, J. Stellbrink, D. Richter, B. Farago, K. Almdal, and R. Faust, Macromolecules 34, 1281 (2001).

[53] B. Frick, D. Richter, and Cl. Ritter, Europhys. Lett. 9, 557 (1989).

[54] J. D. Londono, B. K. Annis, A. Habenschuss, G. D. Smith, O. Borodin, C. Tso, E. T. Hsieh, and A. K. Soper, J. Chem. Phys. 110, 8786 (1999).

[55] M. Misawa, T. Kanaya, and T. Fukunaga, J. Chem. Phys. 94, 8413 (1991).

[56] J. M. Carella, W. W. Graessley, and L. J. Fetters, Macromolecules 17, 2775 (1984).

[57] T. Unruh, C. Smuda, S. Busch, J. Neuhaus, and W. Petry, J. Chem. Phys. 129, 121106 (2008).

[58] D. Richter, B. Farago, R. Butera, L. J. Fetters, J. S. Huang, and B. Ewen, Macromolecules 26, 795 (1993).

[59] The viscosity results from Carella et al. [56] give a somewhat steeper $T$ dependence.

[60] L. J. Fetters, D. J. Lohse, D. Richter, T. A. Witten, and A. Zirkel, Macromolecules 27, 4639 (1994).

[61] M. Rubinstein and R. H. Colby, Polymer Physics (Oxford University Press, Oxford, 2003).

[62] J. T. Padding and W. J. Briels, J. Chem. Phys. 114, 8685 (2001).

[63] We have calculated $S(Q)$ in absolute value $S(Q \rightarrow \infty)=1$ by comparing our differential cross section to that measured by Londono et al. [54] that extends up to $43 \AA^{-1}$.

[64] J. Colmenero and A. Arbe, Macromol. Symp. 121, 133 (1997).

[65] A. Moral, A. Arbe, and J. Colmenero, Physica B 276-278, 440 (2000).

[66] A. Arbe, D. Richter, J. Colmenero, and B. Farago, Phys. Rev. E 54, 3853 (1996).

[67] K. Sköld, Phys. Rev. Lett. 19, 1023 (1967).

[68] A. Arbe, J. Colmenero, and D. Richter, in Broad Band Dielectric Spectroscopy, edited by F. Kremer and A. Schönhals (Springer-Verlag, Heidelberg, 2002), pp. 685-718. 